\title{
Marshall Syndrome or PFAPA (Periodic Fever, Aphthous Stomatitis, Pharyngitis, Adenopathy) Syndrome
}

\author{
MUHBEGUM
}

\begin{abstract}
Summary:
Marshall Syndrome PFAPA (Periodic Fever, Aphthous Stomatitis, Pharyngitis, Adenitis) Syndrome is a chronic condition, typically starting 2-5 years old, in which fever occurs periodically (lasts for 3-7 days), accompanied by aphthous-like ulcers, pharyngitis and/or cervical adenitis. The patients have no clinical symptoms between episodes and it is required to exclude all other diseases before confirming the diagnosis. The dramatic response to treatment with steroid helps diagnosing PFAPA. We are presenting the case of an 8 years male patient, with the history of recurrent episodes of fever (onset at the age of two), oral ulcer and difficulty in deglutition who constantly received antibiotic therapy and or antifungal prescribed by different doctors. Clinically the patient was febrile, mildly pale, cervical lymphadenopathy, aphthous
\end{abstract}

Introduction:

The Marshall Syndrome or PFAPA (Periodic Fever, Aphthous stomatitis, Pharyngitis, and Adenitis) syndrome is a clinical entity that was first recognized by Gary Marshall, Alexander Lawton, and colleagues in $1987^{1}$. It is characterized by an onset before the age of 5 years; quite regularly recurring episodes of fever, with at least one of the three associated constitutional signs of aphthous stomatitis, pharyngitis, or cervical adenitis without any signs of upper respiratory tract infection; completely asymptomatic inter-febrile periods with normal growth and development and the exclusion of cyclic neutropenia, immunodeficiency and auto-inflammatory syndromes ${ }^{2}$. It usually begins between the ages of two and five years and attacks ceases before 10 years of age in most patients ${ }^{3}$. Many treatment have been used with variable results including antibiotics, non-steroid anti-inflammatory drugs, acetylsalicylic acid, colchicine, antiviral medicines, steroids, cimetidine, and tonsillectomy ${ }^{1}$

\footnotetext{
Address of Correspondence: Dr Most. Umme Habiba Begum, Associate Professor, Department of Pediatrics, Rangpur Community Medical College, Rangpur. Mobile: 01712546647, 01521248085, e-mail: uhabibarp27@gmail.com, arafanis@gmail.com Received: 10 August 2014

Accepted: 17 February 2016
}

ulcers in the tongue with inflamed tonsils, covered with thick exudates and pharyngeal wall was inflamed and folliculated. There was just palpable liver. Laboratory investigations performed but it was without serological confirmation. Throat swab culture was negative. The child received steroid (Prednisolone) with favorable outcome. Subsequently, the patient presented with similar episodes of fever which disappeared within 24 hours of single dose of prednisolone. After Ranitidine ( $\mathrm{H}_{2}$ blocker) prophylaxis the patient remains asymptomatic for about one and half years. With our best knowledge this is the first case report of Marshall Syndrome or PFAPA Syndrome in Bangladesh.

Key Words: Marshall syndrome, PFAPA syndrome.

(J Bangladesh Coll Phys Surg 2016; 34: 222-224)

\section{Case report:}

An 8 years old boy reported to out patient department of Northern Private Medical College Hospital, Rangpur with the history of recurrent fever, oral ulcer and difficulty in deglutition since 2 years of age. He experienced the similar attack every 2 to 3 months interval. Each episode was associated with high fever (duration 2 to 3 days) and symptoms persist 7 to 8 days with or without treatment. For last six years his parents took him repeatedly to ENT specialist and other different doctors including village doctors, general practitioners and specialized doctors. In each visit he was prescribed with antibiotics, anti- pyretic, analgesics, and anti-fungal oral gel or antiseptic mouth wash. He was also treated with homeopathic medicine. This scenario created much worry and apprehension to parents about their child. The boy has two other siblings, one brother and a sister, all are healthy. The boy was also worried and depressed about disease. His parents did not suffer from such physical illness.

Clinically, the patient was weighed $16 \mathrm{~kg}$, ill-looking, depressed in mood, febrile (temperature $101^{\circ} \mathrm{F}$ ), mildly pale, non tender discrete cervical (anterior chain) and sub-mandibular lymphadenopathy. Examination of oral cavity and throat showed aphthous ulcers in base and 


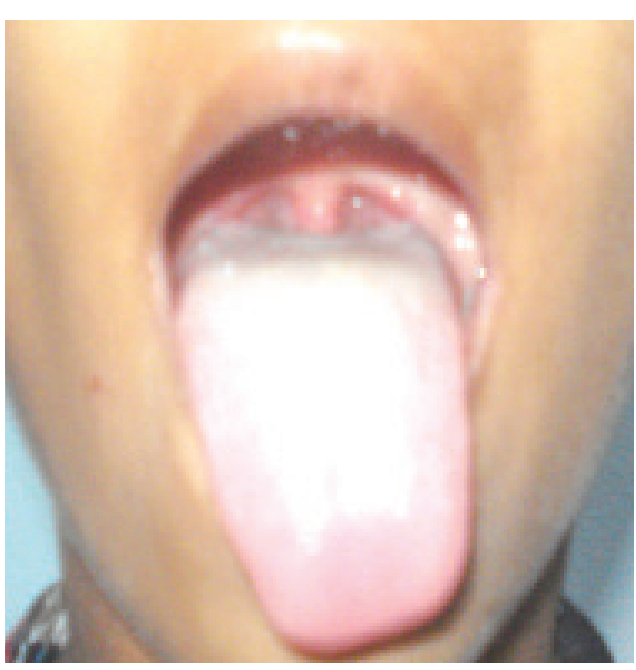

Fig.-1: Enlarged tonsils, aphthous ulcer and erythematous pharynx with exudates.

Table-I

Diagnostic criteria for Marshall/PFAPA syndrome 4 (Source: Thomas et al. 1999 from; www.orpha.net/PFAPA.pdf /January 2004)

Regularly recurring fever with an early age of onset $(<5$ years of age)

Symptoms in the absence of upper respiratory tract infection with at least one of the following clinical signs:

a) aphthous stomatitis

b) cervical lymphadenitis

c) pharyngitis

Exclusion of cyclic neutropenia

Completely asymptomatic interval between episodes

Normal growth and development

margins of tongue; both tonsils were inflamed, enlarged and covered with thick exudates; pharyngeal wall was inflamed and folliculated. There was just palpable liver; no other organomegaly or physical sign. The patient was suspected as Marshall syndrome and investigation was done. Investigations showed, Hemoglobin $-9.4 \mathrm{gm} /$ dl, ESR-36 mm in $1^{\text {st }}$ hour,TLC-10x10\%/L, N-51\%, L-46\%, M- $01 \%$, E- $02 \%$; PBF- RBC series of cells were markedly hypochromic, no evidence of hemolysis, white cells and platelet series were adequate and normal; CRP- $<6.0$ $\mathrm{mg} / \mathrm{dl}$ (Normal- upto $6.0 \mathrm{mg} / \mathrm{dl}$ ). Throat swab culture was negative. Ultrasound of abdomen showed normal finding. He was prescribed with Prednisolone $1.5 \mathrm{mg} / \mathrm{kg}$ per day for five days and Ranitidine $5 \mathrm{mg} / \mathrm{kg}$ per day along with Paracetamol. Patient was followed up on second day and became afebrile on the same day after single dose of steroid therapy. He had almost remission of all other symptoms. After 2 months, patient again presented with similar symptoms and treated only with Prednisolone. The patient also reported after 5 months with similar attack and he was given Ranitidine $\left(\mathrm{H}_{2}\right.$ blocker) prophylaxis. He is symptom free now for about one and half years.

\section{Discussion:}

The PFAPA syndrome is a chronic disease of unknown etiology. The diagnosis of this disease is difficult because none of its clinical symptoms are pathognomic, and there is no specific biologic abnormality. The symptoms may mimic other recurrent fever ${ }^{4}$. It is typically started in young children, in which high fever occurs periodically at intervals of about $3-5$ weeks, frequently accompanied by aphthous ulcers, pharyngitis and/or cervical adenitis ${ }^{5}$. The Marshall/PFAPA syndrome is defined clinically and diagnosis is made by exclusion. It has diagnostic criteria also (Table 1$)^{6}$. The origin of this syndrome is unknown. There is a slight male predominance but no predilection for a particular ethnic or racial group. Familial cases are rare ${ }^{3}$.

In 1987, Marshall et al. reported a previously undescribed periodic fever syndrome of unknown cause in 12 children. These patients presented with febrile episodes that recurred every 2 to 12 weeks. In all cases, the onset of symptoms started before 5 years of age and the fever reached high temperature $\left(40\right.$ to $\left.41^{\circ} \mathrm{C}\right)$ lasting approximately 5 days. Fever was associated with pharyngitis and stomatitis in $75 \%$ cases, cervical reactive adenopathies in $66.6 \%$ cases, and other minor symptoms such as headache, abdominal pain, nausea, vomiting, chills and malaise. None of these children were immunodeficient ${ }^{1}$. In a study, Tasher et al. identified four different patterns in disease evolution. The most common pattern ( $42 \%$ ) was characterized by episodes becoming gradually shorter and less frequent over time. A unique and common course was remissions and relapses, reported in (26\%) children. Remissions lasted up to 3 years. No change in frequency was reported in 
$28 \%$ children. The least common pattern (4\%) was characterized by episodes becoming gradually more frequent over time ${ }^{7}$.

There are no diagnostic tests for PFAPA syndrome. The diagnosis is based on clinical criteria and exclusion of other possible causes of recurrent fever in children. During febrile attacks, patients present elevation of white blood cell (neutrophils) and of acute phase reactants ${ }^{8}$. Normal acute phase reactants are usually found during the inter-febrile periods. Procalcitonin levels, a significant marker of bacterial infections do not usually increase during febrile attacks, and serum levels of immunoglobulins A, G and $\mathrm{M}$ are also normal. The results of urine and blood cultures, chest radiography and liver function test are normal. Only serum IgD level may be slightly high ${ }^{9}$. The episodes are frequently associated with leukocytosis and an elevation in the ESR. The fevers are unresponsive to antibiotics and non-steroid antiinflammatory drugs. The use of oral prednisone controls symptoms but does not prevent the next episode. ${ }^{4,10}$

In our case, symptoms were started in 2 years of age with repeated relapses and short remissions. Patient had typical clinical features of Marshall/PFAPA syndrome. There was no family history, genital ulcer, arthritis, rash, peritonitis or pleurisy. Investigation showed normal finding except high ESR and low hemoglobin. There was dramatic treatment response with prednisolone and remission for about one and half years after Ranitidine $\left(\mathrm{H}_{2}\right.$ blocker) prophylaxis.

\section{Conclusion:}

Marshall Syndrome should be suspected in children with periodic fever associated with pharyngitis, cervical adenitis and aphthous stomatitis. It has a considerable socioeconomic impact on families because of the number of lost school, or working days, and the physical and psychological costs of the disease. Use of oral prednisolone (steroid) and Ranitidine ( $\mathrm{H}_{2}$ blocker) are the therapeutic options currently used. But further studies are needed to identify laboratory markers that would help to identify Marshall / PFAPA Syndrome patients and allow the best therapeutic approach to be defined.

\section{References:}

1. Marshall GS, Edwards KM, Butler J, Lawton AR; Syndrome of periodic fever, pharyngitis, and aphthous stomatitis. J Pediatr. 1987; 110:43-46.

2. Susanna Esposito, Sonia Bianchini, Miriam Fattizzo, Elena Baggi, Paola Marchisio, Donato Rigante.The enigma of periodic fever, aphthous stomatitis, pharyngitis and adenitis syndrome.J Pediatr Infect Dis;. 2014; 33 (6); 650-652

3. Shai Padeh, Brezniak N, Zemer D, Pras E, Livneb A, Langevitz P, et al. Periodic fever with aphthous stomatitis, pharyngitis and adenitis (PFAPA) syndrome; J Pediatr. 1999;135;98-101.

4. Cazeneuve C, Genevieve D, Amselem S, Hentgen V, Hau I, Reinert P. MEFV gene analysis in PFAPA. J Pediatr. 2003;143:140-141.

5. Thomas, Kenneth Tyson; Feder, Lawton, Edwards; Periodic fever syndrome in children; J Pediatr (Retrieved). 2008;135: 1-5.

6. Thomas KT, Feder HM, Lawton AR, Edwards KM; Periodic fever syndrome in children. J Pediatr 1999; $135 ; 15-21$.

7. D Tasher, E Somekh, and I Dalal; PFAPA syndrome: new clinical aspects disclosed. Arch Dis Child. 2006; 91: 981-984.

8. Cecilia Lazea, Rodica Manasia, Calin Lazar. Marshall Syndrome - a challenge in medical practice. Pediatr Croat.2015; 59: 39-43

9. Takao Yoshihara, Imamura T, Yokori K, Mayumi Shibata, Gen Kano, Shinya Osone. Potential use of procalcitonin concentration as a diagnostic marker of the PFAPA syndrome. Eur J Pediatr. 2007; 166;621-622

10. Feder HM Jr. Periodic fever, aphthous stomatitis, pharyngitis,adenitis: a clinical review of a new syndrome. J Pediatr. 2000;12: 253-256. 